\title{
Modification of Airflow Structure Due to Wave Breaking on a Submerged Topography
}

\author{
Petter Vollestad ${ }^{1}$ (D) $\cdot$ Atle Jensen ${ }^{1}$
}

Received: 28 September 2020 / Accepted: 16 May 2021 / Published online: 23 June 2021

(c) The Author(s) 2021

\begin{abstract}
Experimental results from a combined wind-wave tank are presented. Wind profiles and resulting wind-wave spectra are described, and an investigation of the airflow above breaking waves is presented. Monochromatic waves created by the wave maker are directed towards a submerged topography. This causes the waves to break at a predictable location, facilitating particle-image-velocimetry measurements of the airflow above steep breaking and non-breaking waves. We analyze how the breaking state modifies the airflow structure, and in particular the extent of the sheltered area on the leeward side of the waves. Results illustrate that while the geometrical properties of the waves greatly influence the airflow structure on the leeward side of the waves, the state of breaking (i.e., whether the waves are currently in a state of active breaking) is not observed to have a clear effect on the extent of the separated flow region, or on the velocity distribution within the sheltered region.
\end{abstract}

Keywords Airflow separation - Air-sea interaction · Particle image velocimetry $\cdot$ Wave breaking

\section{Introduction}

As air flows over water, energy, and momentum is transferred to the liquid phase, inducing surface waves and currents. The total momentum flux (wind stress) is the sum of tangential shear and normal pressure forces (form drag) at the interface, where the form drag is generally accepted as the dominant contributor to the wind-wave energy flux when a wave field is established (Young 1999). The physical mechanisms responsible for the energy transfer from wind to waves are however not fully understood (Belcher and Hunt 1998; Donelan et al. 2006; Kihara et al. 2007; Sullivan and McWilliams 2010). A unified theory of wave growth is complicated, as the dominant mechanism will vary based on the wind and wave conditions, and as multiple mechanisms are operating at the same time.

One of these mechanisms assessed to be important for steep and breaking waves is airflow separation (Banner 1990; Kudryavtsev and Makin 2001; Donelan et al. 2006; Babanin et al. 2007; Sullivan and McWilliams 2010). The presence of airflow separation over water waves

Petter Vollestad

pettervo@math.uio.no

1 Department of Mathematics, University of Oslo, Oslo, Norway 
has caused some debate in the literature. Banner and Melville (1976) and Gent and Taylor (1977) analyzed monochromatic, quasi-steady waves, and defined airflow separation as "the occurrence, in a frame of reference moving with the waves, of a streamline leaving the water surface" (Gent and Taylor 1977). This requires the surface velocity to match the wave speed $C$, which is the criterion for incipient breaking (Banner and Phillips 1974). Thus, it was concluded that airflow separation over water waves will only occur in conjunction with wave breaking.

However, early flow visualizations of airflow over water waves indicated that intermittent airflow patterns resembling separated flows behind fixed bodies frequently occurred also over non-breaking waves (Kawai 1981; Weissman 1986), and it was argued by Weissman (1986) that the separation criterion of Banner and Melville (1976) and Gent and Taylor (1977) was not appropriate for a moving surface. Airflow separation in a turbulent flow over an unsteady boundary is not easy to define (Simpson 1989; Miron and Vétel 2015; Sullivan et al. 2018; Buckley and Veron 2019), and Buckley and Veron (2019) argued that it is in general not possible to identify separation using velocity measurements of the airflow alone. However, there are features of the separated flow that are detectable and which indicate intermittent flow separation.

Intermittent airflow separation over water waves is characterized by a detachment of the shear layer at the crest, enhanced dissipation downstream of the separation point, and a region of low wind speed below the detached shear layer (Buckley and Veron 2016; Husain et al. 2019). As airflow separation is an intermittent process, phase-averaged flow fields do not show the separated flow structures directly, but increased frequency and intensity of the airflow separation events are observed to enhance the size of the recirculating structure observed on the leeward side of the wave, when the phase-averaged velocity field is seen in a frame of reference moving with the wave speed (Husain et al. 2019; Vollestad et al. 2019). The separated region is associated with a significant pressure drop (Banner 1990; Reul et al. 2008; Sullivan et al. 2018), resulting in a phase shift of the wave-coherent pressure field relative to the wave slope (Donelan et al. 2006; Husain et al. 2019), and enhanced form drag relative to the non-separated case.

While intermittent airflow separation is observed to occur prior to wave breaking (Weissman 1986; Reul et al. 2008; Tian et al. 2010; Sullivan and McWilliams 2010), it is generally accepted that wave breaking induces stronger and more sustained airflow separation (Banner 1990; Babanin et al. 2007; Reul et al. 2008). However, as discussed by Sullivan et al. (2018), "the present understanding of the details of airflow separation over steep waves and its connection to wave breaking is far from complete". Buckley and Veron (2016) analyzed the airflow over both mechanically-generated and wind-generated waves. Airflow separation events (characterized by a detached shear layer) were observed over wind-waves as the equivalent $10-\mathrm{m}$ wind speed was approximately $5 \mathrm{~m} \mathrm{~s}^{-1}$. Wave breaking was however not quantified, and it is concluded by Buckley and Veron (2016) that one of the open questions that remain is "whether wave breaking is the only condition for the separation of turbulent boundary layers over waves".

Banner (1990) investigated the airflow above short, mechanically-generated waves. Tuning the steepness of the incoming waves to generate incipient and actively breaking waves, he found that the steeper breaking waves significantly altered the airflow, and increased the form drag typically by a factor of 2 compared to the non-breaking waves. While the overall wave steepness $a k$ was comparable for the incipient and actively breaking waves, the front-face steepness was significantly higher for the actively breaking waves, and from the results it is not clear whether the spilling region associated with wave breaking, or the enhanced (local) steepness of the actively breaking waves is responsible for the increased form drag. Isolating 
these effects (the geometrical properties of the waves and the state of breaking) is difficult, as breaking will change the wave geometry. Sullivan et al. (2018) performed large-eddy simulations of airflow above wave profiles extracted from the experiments of Banner (1990). By parameter tuning the simulated wave profiles and the enhanced surface drift associated with wave breaking, Sullivan et al. found that the wave steepness, not the state of breaking, dictates the form drag of the waves. This is an important result for modelling airflow above waves, as the complex flow structure associated with wave breaking represents a significant modelling challenge. While Sullivan et al. (2018) used a modified one-fluid approach, Yang et al. (2018) used a two-fluid direct numerical simulation approach to simulate the airflow over breaking waves. Results indicate that the transition from non-breaking to actively spilling breaking does not significantly modify the airflow above the waves, or the momentum transfer between the phases. Plunging breakers were, on the other, hand seen to significantly modify the airflow structure, and to cause a momentum flux from the water to air, as a high pressure zone formed in front of the plunging crest as it accelerated forwards.

Reul et al. (2008) investigated the airflow over short breaking waves, generated by focusing wave packets. Airflow separation was observed using particle image velocimetry (PIV) both prior to and during the wave breaking process, and the features of the separated flow region were found to be similar to the flow over a backwards-facing step. Both the horizontal and vertical extent of the separated flow region was found to be well correlated with the crest front-face steepness $\varepsilon_{\text {crest }}$ ( $\Delta y / \Delta x$ from crest to zero-down crossing on leeward side of the wave). While the evolution of the airflow field over single waves transitioning to breaking was presented, indicating that active wave breaking enhanced the airflow separation, no statistical analysis was performed to isolate the effect of the spilling region from the geometrical properties of the waves. By measuring the pressure some distance above the wave crests, Reul et al. (2008) also demonstrated how airflow separation drastically reduces the pressure within the separated zone, enhancing the form drag of the waves.

Donelan et al. (2006) performed field experiments measuring the pressure field correlation with wave slope. A phenomenon of 'full separation' was observed for strongly wind-forced waves, where the airflow detached from the crest and reattached on the windward side of the downstream wave. In this situation, the magnitude of the wave-coherent pressure fluctuations is reduced, as the airflow does not 'see' the wave profile (Donelan et al. 2006). In a later paper analyzing the same experimental results, Babanin et al. (2007) detected breaking waves based on acoustic measurements. The form drag from breaking waves was observed to be significantly increased compared with non-breaking waves, implying that wave breaking enhances flow separation. The effect of wave steepness was isolated by considering breaking and non-breaking waves at the same crest rear face (windward side) steepness. Results from Reul et al. (2008) do however indicate that the crest front-face steepness is the more relevant property in relating the instantaneous wave geometry to the extent of the separated zone.

Analyzing microscale breaking waves in a stratified pipe flow set-up, Vollestad et al. (2019) found that for a moderate wind speed, active wave breaking had the effect of reducing the sheltered region, compared with waves at similar crest front-face steepness. This was attributed to a reduction in the extent and/or frequency of airflow separation. At higher gas flow rates, the geometrical properties of the waves $\left(\varepsilon_{\text {crest }}\right)$ was seen to dominate the development of the airflow structure.

In the present work, a PIV analysis of the airflow above actively breaking and nonbreaking waves is performed. Waves are generated by a mechanical wave maker and allowed to propagate under the action of wind. As the waves propagate above a submerged structure, wave breaking is induced (depending on the incoming wave characteristics). Wave breaking over submerged structures has been investigated previously (Smith and Kraus 1990, 1991; 


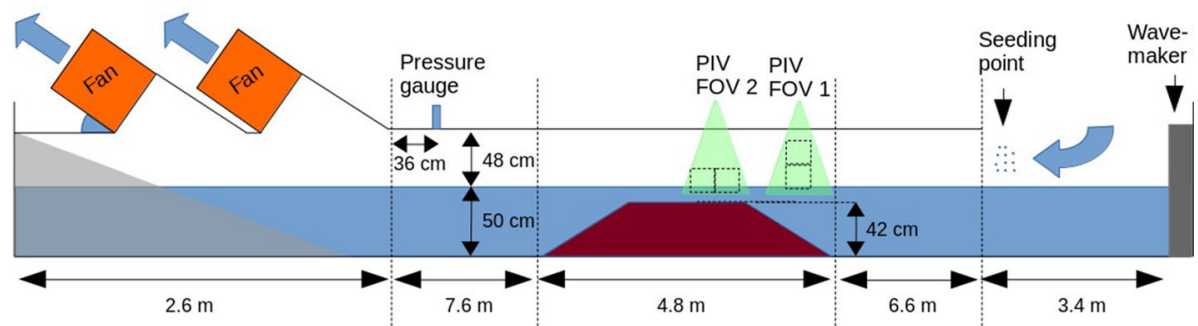

Fig. 1 Schematic view of experimental set-up. Note that FOV refers to the field-of-view

Johnson 2006; Blenkinsopp and Chaplin 2008; Yao et al. 2013), and is known to be highly dependent on the water level over the structure, and to differ in characteristics from wave breaking both on beaches and in the open ocean. Full details of the wave-breaking process are outside the scope of the present work, but the visual characteristics of the waves are used to classify the waves as either breaking or non-breaking. For the spilling-type breakers observed, active wave breaking could be identified by surface disturbances on the leeward side, which evolved into a distinct spilling motion, causing air entertainment and splashup of water droplets for the more severe breaking cases. Focus is on the airflow above the waves, and the area sheltered due to non-separated or separated sheltering is quantified for both breaking and non-breaking waves, to examine whether there are signs of enhanced (or reduced) airflow separation comparing breaking and non-breaking waves at similar steepness. The novelty lies in the simultaneous classification of independent waves as either breaking or non-breaking and evaluation of the sheltered area. Combined with an evaluation of the instantaneous crest front-face steepness, this should clarify whether the state of breaking and an active spilling region significantly influences the airflow over waves, or if the geometrical properties govern airflow separation and sheltering.

\section{Experimental Set-up}

The experiments were conducted in the large wave tank at the University of Oslo. The wave tank is $25 \mathrm{~m}$ long and $0.5 \mathrm{~m}$ wide. Waves are generated by an hydraulic piston wave maker in one end, and a wave damping beach constructed by wire-mesh is installed in the opposite end of the tank; $10 \mathrm{~m}$ from the wave maker, a submerged sloping step is installed. The step is used to induce regular wave breaking at a fixed location, enabling PIV investigation of waves transitioning to active breaking. An overview of the experimental set-up is presented in Fig. 1. Details of the submerged step are described in Fig. 2.

For the present study, the wave tank has been modified to facilitate a wind field above the water surface. Airflow is provided by two construction fans (Heylo FD4000, each with an adjustable flow rate from 0.8 to $1.15 \mathrm{~m}^{3} \mathrm{~s}^{-1}$, when used as stand-alone), located on the downstream end of the tank, which suck air through the tank. Rigid plates are placed on top of the tank, $0.98 \mathrm{~m}$ above the bottom. These are placed along the tank, with a $3.4 \mathrm{~m}$ opening towards the wave maker, where air is allowed to enter. It should be noted that no convergent nozzle or honeycomb flow straighteners were applied to the airflow at the entrance. This simplified the experimental set-up, and the large opening at the inlet was deemed to provide a reasonable wind field as the velocity profile evaluated above the step was found to be close to logarithmic (see Sect. 3). In the experiments performed, the mean water level is kept at 


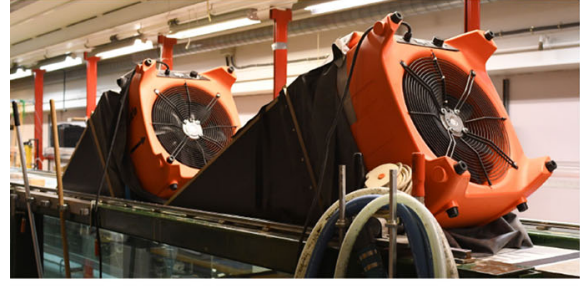

(a)

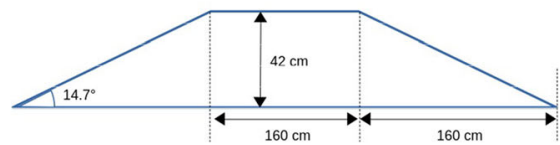

(b)

Fig. 2 a Image of fans used to generate wind field. b Schematic view of the step

$0.5 \mathrm{~m}$, leaving a $0.48 \mathrm{~m}$ air gap above the mean water level, and $0.08 \mathrm{~m}$ of water above the flat section of the submerged step.

Air velocity measurements are performed by the use of PIV in the centre-plane of the wave tank. Two different fields-of-view are used in the present study. While a vertical arrangement before the step is used to evaluate the velocity profiles in Sect. 3 (PIV FOV 1 in Fig. 1, where the centre of the fields-of-view is $10.75 \mathrm{~m}$ from the paddle), a horizontal arrangement above the step (PIV FOV 2 in Fig. 1, centre of the field-of-view is $0.35 \mathrm{~m}$ from the leading edge of the step) is applied for measurements of airflow over steep breaking and non-breaking waves in Sect. 4. In PIV FOV 1, the cameras were tilted downwards at an angle of $3^{\circ}$, while measurements of airflow over breaking waves in PIV FOV 2 required a larger downward looking angle of $11.3^{\circ}$ to fully visualize the centre-plane of the incoming waves. Prior to the experiments, cubic coordinate transforms (from pixel to world coordinates) were created by imaging a grid of $10 \mathrm{~mm} \times 10 \mathrm{~mm}$ spaced dots in the centre-plane of the flume.

Two high-speed cameras (Photron, WX100, Tokyo, Japan) with $50 \mathrm{~mm}$ lenses were used, each providing a field-of-view of approximately $0.18 \mathrm{~m} \times 0.18 \mathrm{~m}$ and a resolution of $2048 \times$ 2048 pixels. The air phase was seeded with small water droplets generated from a high pressure atomizer (custom model manufactured by SCITEK Consultants, Derby, UK). According to the manufacturer, $72 \%$ of the droplets are smaller than $6 \mu \mathrm{m}$. The centre-plane was illuminated by a $147 \mathrm{~mJ}$ ND:YAG double pulsed laser (Quantel, Big Sky Laser, USA), with the laser optics mounted $0.7 \mathrm{~m}$ above the mean water level. This generated a thin light sheet covering the field-of-view of both cameras. While the cameras are capable of 1000 frames per second (fps) at the maximum resolution, the laser has a maximum frequency of $16 \mathrm{~Hz}$. For this reason, the cameras were set to acquire images at a rate of 30 frames per sec, and a frame straddling technique was employed to control the effective $\Delta t$ between an image pair used for PIV. Hence, 15 velocity fields were acquired per second; $\Delta t$ was varied between 150 and $350 \mu$ s depending on the air velocity in the flume. The images were processed in Digiflow by Dalziel Research partners, using a cascade of sub-window sizes with a final sub-window size of $80 \times 80$ pixels, with $50 \%$ overlap for the velocity profiles presented in Sect. 3, and 64 $\times 64$ pixels, with $50 \%$ overlap for the results presented in Sect. 4 . This translates to a spatial resolution of approximately $3.5 \times 3.5$ and $2.7 \times 2.7 \mathrm{~mm}^{2}$ for the processed data respectively. Applying a Gaussian sub-pixel estimator (typical accuracy of 0.1 pixels), and considering that the peak particle displacement was in the range of 15 pixels $/ \Delta t$, the minimum detectable velocity is estimated to be $<1 \%$ of the peak speed observed for each experimental case.

Ultrasonic wave probes (General Acoustics, Ultralab USS 02/HFP, Kiel, Germany) were used to measure the surface elevation in the centreline at $250 \mathrm{~Hz}$. Measurements were performed at the centre location of PIV FOV 1 . Note that PIV and surface elevation measurements were not performed simultaneously, as the ultrasonic wave probes had to be placed within 
the closed air channel, and would affect the airflow (and hence the PIV results), and obscure the incoming laser light if placed at the same streamwise location as the PIV measurements.

The pressure relative to the ambient is evaluated $0.36 \mathrm{~m}$ before the first of the two fans by the use of a smart LD301 differential pressure gauge. By matching the mean water level and underpressure recorded, the velocity profiles were found to be reproducible, and this was used to replicate the air velocity profiles during different stages of the experimental work.

\section{Wind Profiles and Resulting Wind-Wave Spectra}

Particle image velocimetry and surface elevation measurements are performed at the PIV FOV location 1. For the PIV measurements, 600 image pairs were acquired for each camera per experimental run, while the waveprobes were logging each experimental case for $8 \mathrm{~min}$.

The two fans could be operated individually, and by placing a thin latex cover over one of the fans, this could be closed off, not allowing air to be circulated through the fan. This provides some flexibility in the air flow generated. Five experimental cases with increasing wind speed were considered, referred to as wind cases 1 to 5 . Results for the mean wind profiles (at some distance above the mean water level) are presented in Fig. 3, while the spectra of the wind-waves recorded by the ultrasonic probes are presented in Fig. 4. In Fig. 3a, the horizontal velocity is seen to increase steadily for the wind cases considered, from a peak velocity $\left(U_{\max }\right)$ of approximately 3 to $6.2 \mathrm{~m} \mathrm{~s}^{-1}$. Figure $3 \mathrm{~b}$ presents the mean vertical velocity profile, divided by the peak horizontal velocity. For all cases considered, the vertical velocity is seen to be directed downwards, with increasing intensity towards the interface. Considering turbulent flow in non-circular channels, it is well known that secondary flows (mean flow structures perpendicular to the axial flow direction) are generated (Prandtl 1927; Speziale 1982). For fully developed single-phase flow through a square duct (closely resembling the cross-section experienced by the gas in the present work), secondary flows are observed to be directed from the centre to the corners along the corner half-angle, out along the walls towards the midpoint between the corners, and up towards the duct centre. Thus, we would expect a flow in the positive vertical direction at the duct centreline, from the interface to the centre of the duct. Roughness variations around the circumference are also known to drastically influence the secondary flow patterns, leading to a flow down towards the rough region (Hinze 1967, 1973). As the water surface is significantly 'rougher' than the surrounding walls, this is a possible explanation for the observed flow pattern. It is also considered likely that the secondary flows are generated at the inlet, as air is sucked into the tank through an opening of the flume roof, creating a downwards directed wind field at the inlet which can persist to the measurement section. Regardless of the origin, these non-zero vertical velocity profiles represent a source of uncertainty when comparing results from the wind-wave flume with airflow over waves in the ocean.

The frequency spectra presented in Fig. 4 illustrate that the peak frequency is reduced, and the energy of the wave field is increased with increasing wind speed. A significant increase in the wave field energy is observed from wind cases 1 to 2 . This is consistent with visual observations of the wave-field development.

Figure 3 presents the velocity profiles in a semi-logarithmic plot. For the higher wind speeds, the velocity profiles some distance above the waves are found to be well represented by the classical logarithmic profile, which can be expressed as

$$
u=\frac{u_{*}}{\kappa} \ln \left(y / y_{0}\right),
$$




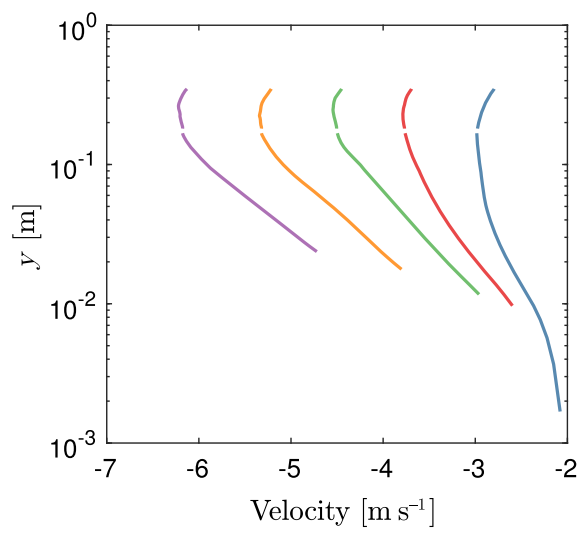

(a)

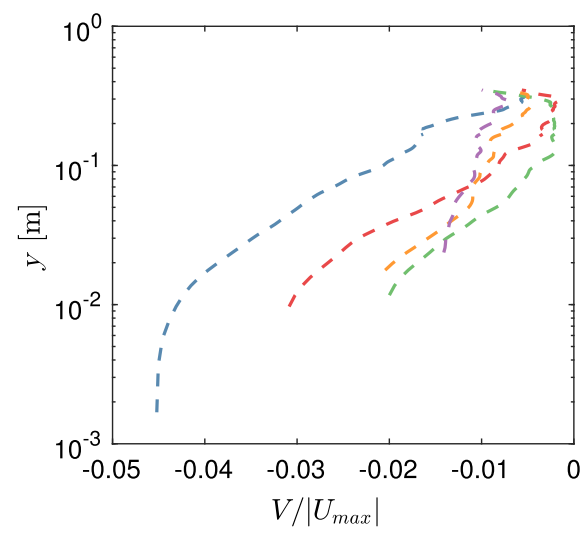

(b)

Fig. 3 Mean flow velocity profiles at PIV FOV 1. Plotted from $y=3 \cdot \eta_{r m s}$. a Mean horizontal velocity. $\mathbf{b}$ Mean vertical velocity divided by maximum horizontal velocity

Fig. 4 Wave probe power spectral density $(P S D)$ for the wind-wave cases under investigation. Wave probe located at centre of PIV FOV 1

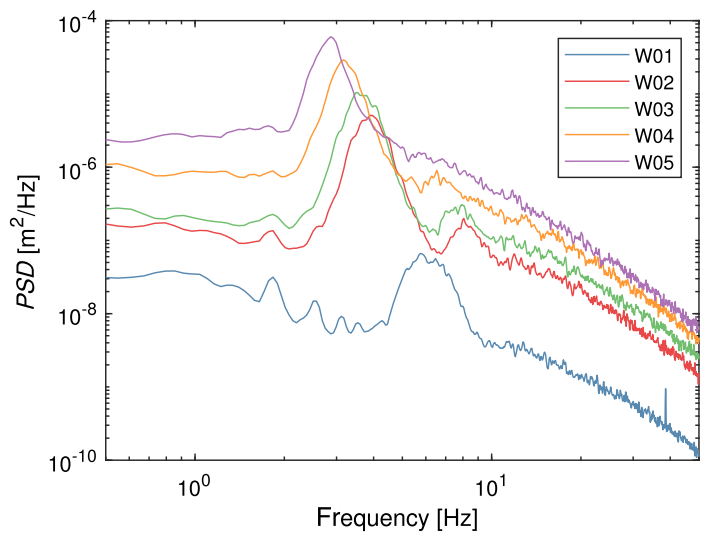

where $u$ is the longitudinal (horizontal) velocity component, $u_{*}$ is the air friction velocity, $\kappa$ is the Von Kármán constant (set equal to 0.41) and $y_{0}$ is the roughness length. Equation 1 was fitted to a part of the velocity profile exhibiting a logarithmic behaviour, deducing $u_{*}$ and $y_{0}$. The logarithmic profile was then used to estimate an equivalent $U_{10}$ (mean velocity evaluated $10 \mathrm{~m}$ above the surface). Results are presented in Table 1, together with the peak horizontal velocity recorded $\left(U_{\max }\right)$, the peak frequency of the spectra $\left(f_{p}\right)$, and the root-mean-square (r.m.s.) surface elevation recorded $\left(\eta_{r m s}\right)$. It should be noted that for the lowest wind speed considered, a distinct logarithmic velocity profile is not observed as for the three highest wind speeds. Values obtained by fitting Eq. 1 to the narrow region between 0.02 and 0.05 $\mathrm{m}$ above the mean water level are presented in Table 1, but should be considered with care. Deviations from the logarithmic velocity profile for this experimental case may be linked to the significant vertical velocity component observed for the same case. 
Table 1 Results from the wind profile and wave gauge analysis

\begin{tabular}{lllllll}
\hline Wind case & $U_{\max }\left(\mathrm{m} \mathrm{s}^{-1}\right)$ & $u_{*}\left(\mathrm{~m} \mathrm{~s}^{-1}\right)$ & $y_{0}(\mathrm{~mm})$ & $U_{10}\left(\mathrm{~m} \mathrm{~s}^{-1}\right)$ & $\eta_{r m s}(\mathrm{~mm})$ & $f_{p}(\mathrm{~Hz})$ \\
\hline 1 & 2.98 & 0.103 & 0.0051 & 4.23 & 0.54 & 5.80 \\
2 & 3.76 & 0.189 & 0.0293 & 5.86 & 2.66 & 3.96 \\
3 & 4.55 & 0.249 & 0.0865 & 7.08 & 3.62 & 3.54 \\
4 & 5.34 & 0.303 & 0.1040 & 8.47 & 5.48 & 3.17 \\
5 & 6.22 & 0.344 & 0.0850 & 9.80 & 7.66 & 2.87 \\
\hline
\end{tabular}

\section{Airflow over mechanically-generated Breaking and Non-breaking Waves}

In this section, the airflow above mechanically-generated waves travelling over the submerged step is analyzed.

\subsection{Wave Case Description}

Monochromatic waves were generated by the wave paddle. The wave frequency was set to $1.5 \mathrm{~Hz}$, and five different amplitudes are analyzed. These are referred to as wave cases 1 to 5 . To characterize the waves generated by the paddle, these were measured without the influence of wind, and without the submerged step using ultrasonic wave probes at the centre of PIV FOV 1.

An extract of the surface elevation measurement for the intermediate amplitude case is presented in Fig. 5a. In Fig. 5b, the spectra of the surface elevation measurements of the same case are presented and compared with the spectra from a deep water third-order Stokes wave (theoretical third-order Stokes wave, see, e.g., Grue et al. 2003, sampled at the same frequency as the wave probes, and processed in the same manner as the experimental results). The spectra were evaluated using the Welch method, which is a periodogram estimate of the power spectral density, based on averaging the discrete Fourier transform of multiple extracts of the time series. The comparison illustrates that the second and third harmonics are measured with reasonable accuracy. From the measurements we also observe higher order harmonics. However, these were observed to increase in amplitude, indicating that the measurements are influenced by noise.

The input frequency and mean crest height $\left(\overline{\eta_{c}}\right)$ measured was used to calculate the wave steepness $a k$ using the third-order Stokes equation for deep waters, according to (Grue et al. 2003)

$$
\eta_{c}=a\left(1+\frac{1}{2} a k+\frac{1}{2}(a k)^{2}\right),
$$

in combination with the non-linear dispersion relation

$$
\omega=\sqrt{g k\left(1+(a k)^{2}\right)},
$$

where $\omega, g, k$ and $a$ are the angular frequency, acceleration of gravity, wavenumber, and amplitude of the wave, respectively. The deep water approximation was justified as $\tanh (k h) \approx 0.9997$ for the incoming waves considered. The wave phase speed was evaluated according to $C=\sqrt{\left(1+(a k)^{2}\right)(g / k)}$. Results presented in Table 2 illustrate that the wave 


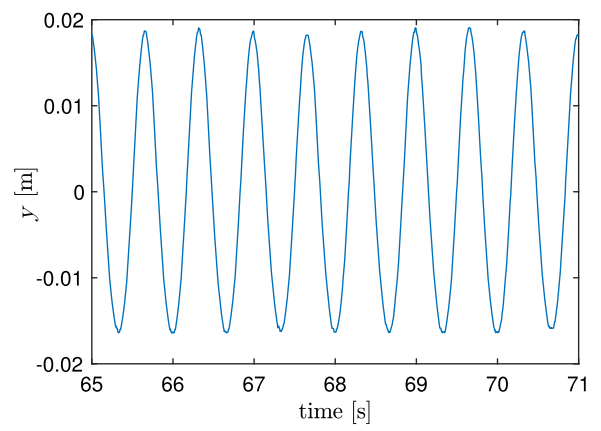

(a)

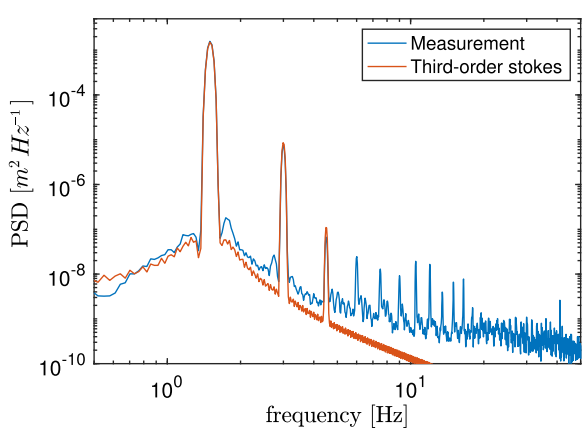

(b)

Fig. 5 a Raw data from wave probe measurements of incoming wave, wave case 3. b Wave probe spectra of the same case compared with third-order Stokes theory

Table 2 Results from the analysis of the incoming waves generated by the paddle

\begin{tabular}{llllll}
\hline Wave case & Frequency $(\mathrm{Hz})$ & $\overline{\eta_{c}}(\mathrm{~mm})$ & $\bar{H}(\mathrm{~mm})$ & $a k[-]$ & $C\left(\mathrm{~m} \mathrm{~s}^{-1}\right)$ \\
\hline 1 & 1.5 & 10.2 & 20.4 & 0.09 & 1.05 \\
2 & 1.5 & 15.1 & 28.2 & 0.13 & 1.05 \\
3 & 1.5 & 19.2 & 35.1 & 0.16 & 1.06 \\
4 & 1.5 & 23.3 & 42.1 & 0.18 & 1.07 \\
5 & 1.5 & 27.9 & 50.3 & 0.21 & 1.08 \\
\hline
\end{tabular}

steepness increases from 0.09 to 0.21 for the cases considered. Also presented in Table 2 is $\bar{H}$, the mean wave height recorded.

The five wave cases presented in Table 2 were combined with wind cases 1, 3 and 5 presented in Table 1 for a total of 15 experimental cases of mechanically-generated waves passing over the step under the influence of wind. Wave breaking on beaches is typically categorized as either spilling, plunging, collapsing or surging (Galvin 1968). However, analyzing monochromatic waves over a submerged sloping reef, Yao et al. (2013) found that the breaking events could be classified as either spilling or plunging, where plunging breakers occurred upstream of the reef edge for large-amplitude incoming waves. An overturning crest characteristic of plunging breakers could not be clearly identified from the experimental cases considered, and all breaking events are referred to as spilling. There was however a distinct evolution of the breaking severity with increasing wind input/incoming wave amplitude. A visual inspection of the waves as they passed the step was performed to characterize the cases according to the breaking severity. The categories used are the following:

- No breaking.

- Gentle spilling: little or no visible air entrainment.

- Spilling: considerable air entrainment and some water droplets separating from the interface.

- Intense spilling: splash-up of water droplet above the crest height, and a transition to plunging breakers.

Within the same experimental case, some waves could be observed to be clearly spilling, while others did not break. In Table 3, each experimental case is classified according to the most severe breaking event observed. Hence, for cases classified as 'No breaking', no waves 
Table 3 Classification of wind-paddle cases according to breaking severity at step

\begin{tabular}{|c|c|c|c|c|c|c|}
\hline \multirow[t]{2}{*}{ Wave case } & \multicolumn{2}{|l|}{ Wind case } & & & & \\
\hline & Wind case 1 & & \multicolumn{2}{|l|}{ Wind case 3} & \multicolumn{2}{|l|}{ Wind case 5} \\
\hline 1 & No breaking & (24.4) & No breaking & $(28.0)$ & Gentle spilling & $(34.2)$ \\
\hline 2 & No breaking & (30.9) & Gentle spilling & $(36.0)$ & Gentle spilling & $(42.5)$ \\
\hline 3 & Gentle spilling & (38.3) & Gentle spilling & (42.5) & Spilling & $(49.8)$ \\
\hline 4 & Spilling & (44.0) & Spilling & (47.6) & Spilling & $(56.2)$ \\
\hline 5 & Spilling & (53.7) & Spilling & $(57.6)$ & Intense spilling & $(67.0)$ \\
\hline
\end{tabular}

Mean wave height recorded at PIV FOV $1(\bar{H})$ presented in parenthesis (in mm)

were observed to break, while for the 'Gentle spilling' cases, a combination of gentle spilling and non-breaking waves were typically observed. Results illustrate that the wind plays an important role in the breaking onset at the step, as waves are observed to break for significantly lower amplitude paddle generated waves considering the higher wind speeds. Also presented in Table 3 is the mean wave height $(\bar{H})$, recorded at PIV FOV 1, clearly illustrating the energy input from wind to waves responsible for the enhanced breaking severity observed.

Considering the lowest wind-speed case, the first signs of wave breaking were observed for wave case 3. Similar observations were made without wind. This is consistent with the results from Yao et al. (2013), who found that wave breaking over a submerged reef (considering monochromatic waves without the presence of wind) was initiated when the ratio of the water level at the step and the incoming wave height was less than 2.8, which for the present experimental set-up implies $H>28.6 \mathrm{~mm}$. As seen in Table 2, this is fulfilled for wave cases 3, 4 and 5 without wind. In the presence of wind, the criterion by Yao et al. (2013) is seen to be applicable, except for one case (wind case 1, wave case 2), where no breaking was observed despite a slightly greater incoming wave height.

Figure 6a presents the development of the power spectral density at a constant wind speed (wind case 3) for the different paddle cases, while Fig. 6b illustrates how the spectra evolves with increasing wind speed (considering the intermediate paddle case). For the lowest-amplitude incoming wave, considering wind case 3, a significant increase in the energy of the wave field is observed in the frequency range $3-4 \mathrm{~Hz}$, corresponding to the location of the peak frequency recorded for the pure wind cases (see Fig. 4). This indicates that the wave field can be seen as a superposition of the paddle generated and wind induced wave field for this experimental case. For the higher amplitude paddle cases, no significant increase in energy is observed at the frequencies corresponding to the peak frequency observed in Fig. 4. From Fig. 6b, it is interesting to note that while higher-order harmonics (above the third harmonic) are clearly visible without the influence of wind, these appear to be damped as the wind speed is increased. As previously discussed, measurements of these low-energy bound waves are susceptible to measurement noise, and while the results should be analyzed with care, they indicate that the wind can dampen higher order harmonics of the wave field.

It should be noted that the paddle-generated waves will also influence the airflow, and the computed values for the logarithmic velocity profile presented in Table 1. Wave steepening and breaking at the step will also locally influence the airflow. The process is (due to enhanced form drag over steep and breaking waves) assessed to increase the local momentum flux, and friction velocity. A detailed analysis of the modifications to the logarithmic wind profile due to the mechanically generated waves, and their breaking over the step is outside the scope of the present work. However, the mean velocity at the top of the horizontal field-of-view 


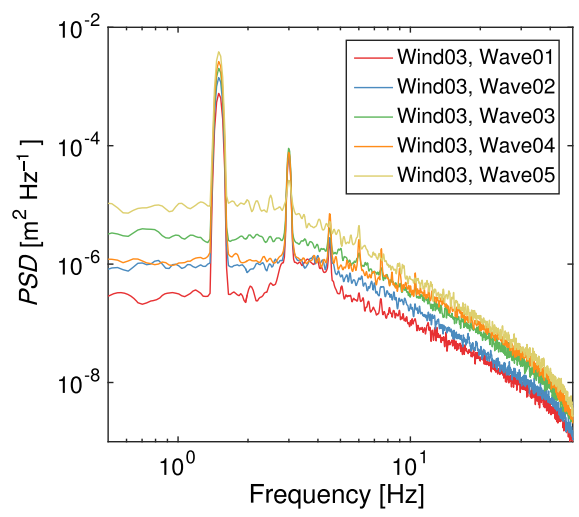

(a)

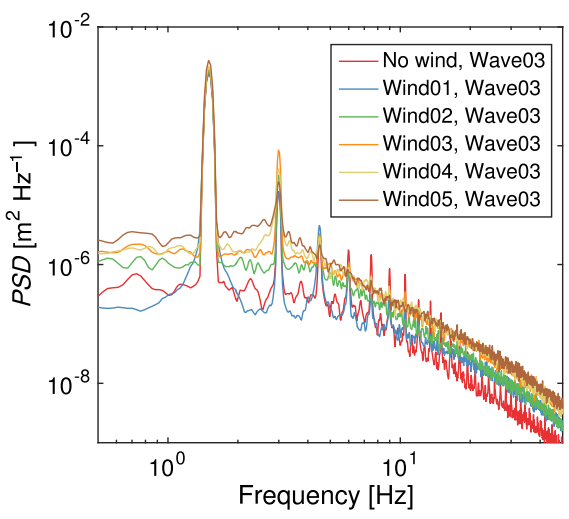

(b)

Fig. 6 Power spectral density ( $P S D$ ) of surface elevation at PIV FOV location 1, considering constant wind speed (wind case 3) (a) and constant paddle-generated wave amplitude (wave case 3) (b)

Fig. 7 Mean horizontal velocity at $y=0.14 \mathrm{~m}$ for the three wind cases considered. Comparison of results without mechanically-generated waves (along $x$-axis), and for the different mechanically-generated wave cases (along $y$-axis)

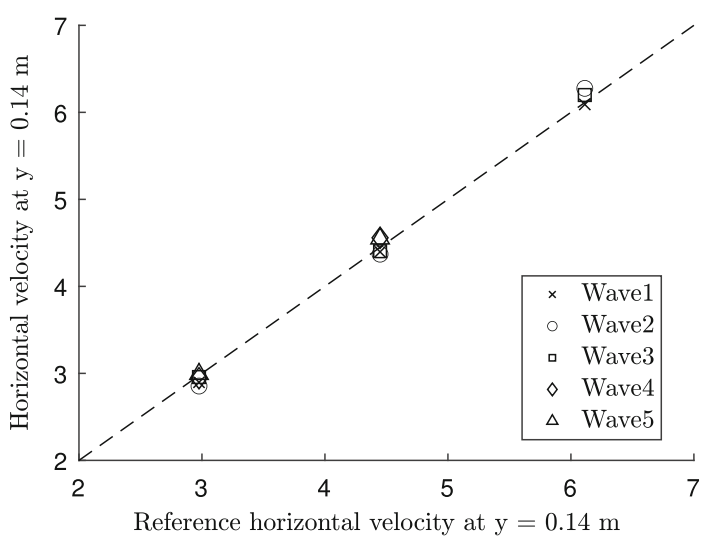

used to assess waves at the step (PIV FOV 2) is compared with the mean velocity at the same height presented in Sect. 3. Results presented in Fig. 7 illustrate that while the mechanicallygenerated waves will modify the wind field close to the interface, the air velocity some distance above the waves is relatively unaffected. Hence, the peak velocity for each wind case, shown in Table 1, represents a characteristic freestream velocity for the wind cases considered, also under the action of mechanically-generated waves.

\subsection{Velocity Fields Over Steep and Breaking Waves}

In this section, PIV results of airflow over steep breaking and non-breaking waves at the step (PIV FOV 2) are presented. The cases (wind/paddle combinations) presented in Table 3 are analyzed. For the two highest paddle amplitudes (wave cases 4 and 5) considering the highest wind speed (wind case 5), intense light reflections (due to the breaking) caused unreliable results, and for this reason these case are omitted from further analysis. Due to light reflections caused by the spilling region, and droplets torn off the wave crests (for the most severe breaking cases considered), accurate automatic detection of the interface (by means 
of detecting gradients of the light intensity) was difficult, and was not assessed to be robust. Thus, the interface was detected manually for all the processed images. While this provides a robust identification of the interface, it limits the number of waves under investigation for each experimental case.

\subsubsection{Time Development of Breaking Waves}

As the PIV system captured the velocity fields at a rate of $15 \mathrm{~Hz}$, a wave crest passing the PIV measurement section was observed for 6-7 images. Figures 8 and 9 illustrate the time development of two waves passing the PIV measurement section. Figure 8 considers the lowest wind-speed case and wave case 3, while Fig. 9 illustrates a wave at the intermediate wind speed and maximum incoming wave amplitude. The instantaneous velocity fields from the two cameras are combined and overlaid the raw PIV images (converted to world coordinates by applying the coordinate transform). Velocity vectors are shown in red, while contours of vorticity are presented in white.

At the first image (time $t_{0}$ ), the waves are assessed to be at early stages of the breaking process, as a distinct bulge and surface disturbances are observed near the wave crests. As the waves pass the PIV section, the breaking is observed to evolve, as an increasingly turbulent spilling region is formed in front of the crest, and as the wave amplitude is reduced. For both waves considered, the shear layer is seen to be detached on the leeward side of the crest during the breaking process. When the wave crest approaches the centre of the field-of-view, the detached shear layer is not fully visible. For this reason, it was difficult to assess the full development of the separated shear layer during the wave breaking process for individual waves. In Fig. 9a the flow appears to attach at $x \approx 0$, and slightly downwind a burst of air is seen to lift off. Such 'blowing up' of the airflow downwind of the reattachment point was also observed by Reul et al. (2008).

It should be noted that significant differences in the time-development of the airflow was observed within each experimental case. While Figs. 8 and 9 can give the impression that the intensity of the separation is increased as the wave breaking intensified, examples of the opposite behaviour was also seen within the experimental results. Wave breaking and airflow separation are clearly highly intermittent processes, and a statistical approach is required to investigate the relationship between them.

\subsubsection{Analysis of Sheltering for Breaking and Non-breaking Waves}

In this section we perform an analysis of the extent of the sheltered region on the leeward side of the crest. Reul et al. (2008) calculated the area bounded by the interface and the instantaneous separating streamline (defined as the position of maximum vorticity above the interface, from detachment to reattachment of the shear layer) divided by the wavelength as a measure of the mean height of the separated flow region. This mean height of the separated shear layer was seen to be well correlated with the crest front-face steepness $\varepsilon_{\text {crest }}$ (defined as $|\Delta y / \Delta x|$ from the crest to the zero-down crossing, i.e., where the wave profile crosses the mean water level on the leeward side of the crest). We perform a similar analysis, but additionally, waves are separated into breaking or non-breaking to investigate if the state of breaking also influences the extent of the sheltered region.

Vollestad et al. (2019) considered the airflow above waves in stratified two-phase pipe flow. The area on the leeward side of the crest where $u<C_{p}$, divided by the length of the leeward side region of interest was evaluated as a measure of the mean height of the sheltered 


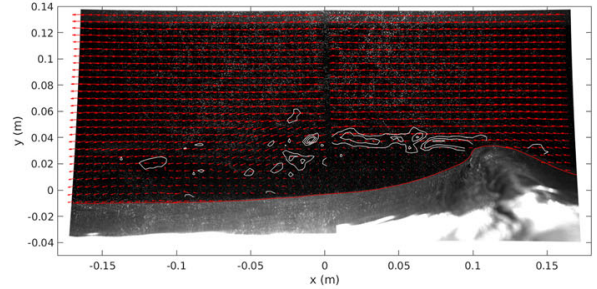

(a)

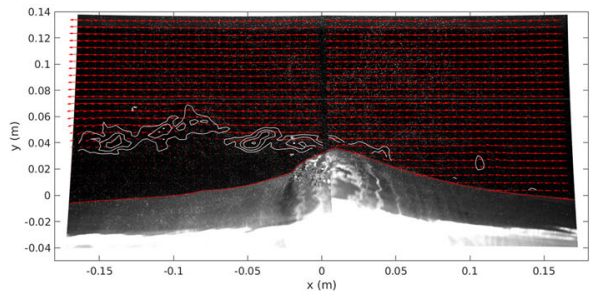

(c)

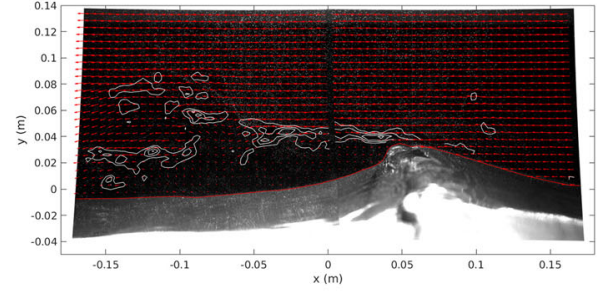

(b)

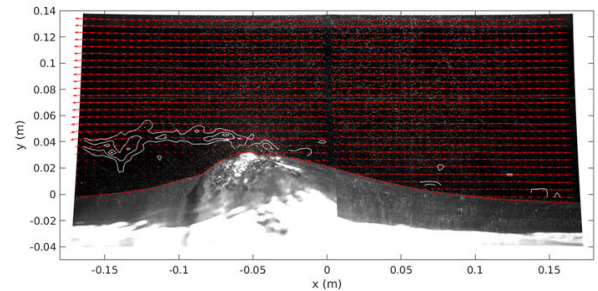

(d)

Fig. 8 Time development of a single wave passing the PIV measurement section. Wind case 1, wave case 3 . Showing $1 / 2$ vectors. White contours: Contours of positive vorticity, separated by $100 \mathrm{~s}^{-1}$. a) $t_{0}$, b) $t_{0}+\Delta t$, c) $t_{0}+2 \Delta t$, d) $t_{0}+3 \Delta t$

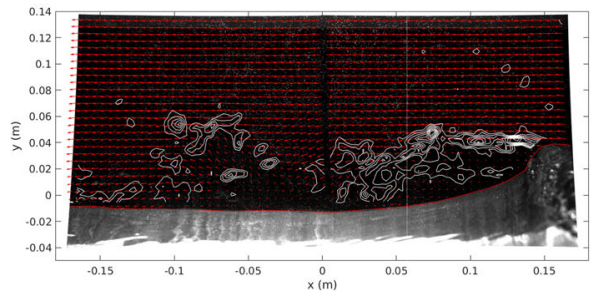

(a)

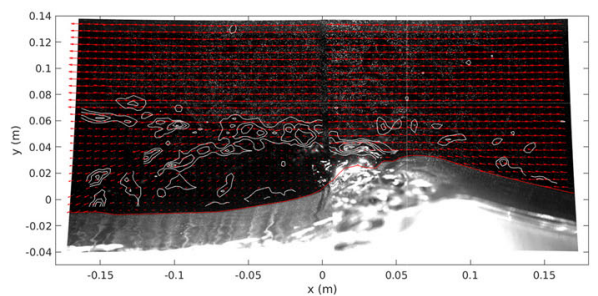

(c)

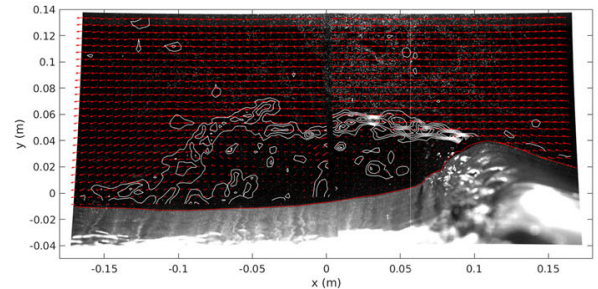

(b)

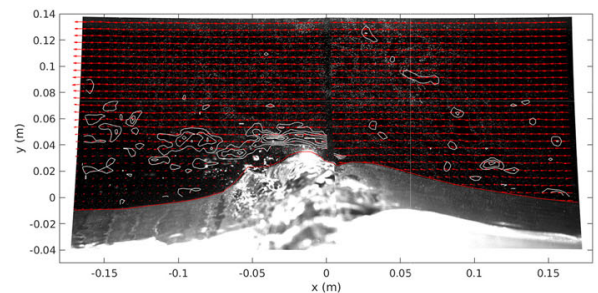

(d)

Fig. 9 Time development of a single wave passing the PIV measurement section. Wind case 3, wave case 5 . Showing $1 / 2$ vectors. White contours: Contours of positive vorticity, separated by $100 \mathrm{~s}^{-1}$. a) $t_{0}$, b) $t_{0}+\Delta t$, c) $t_{0}+2 \Delta t$, d) $t_{0}+3 \Delta t$ 
region. Similar to the results by Reul et al. (2008), this was seen to be well correlated with the front-face steepness. Vollestad et al. (2019) separated the waves into breaking or nonbreaking, employing a threshold for the r.m.s. vorticity in the liquid phase to distinguish actively breaking and non-breaking waves. For the lowest gas flow rate considered, a clear reduction in the mean height of the sheltered region, comparing waves at similar front-face steepness was observed. A similar trend was not seen when considering higher air velocities.

As the waves cross the step, the phase speed is reduced due to shoaling. For all wind/wave combinations under investigation, the crest propagation velocity was evaluated by manually identifying the wave crest in successive images as the waves propagated across the field-ofview. However, for many of the waves under investigation, estimation of the instantaneous crest speed was assessed to be unreliable, as it was difficult to identify the crest location both for wind ruffled waves of gentle slopes and for waves undergoing strong breaking. For this reason, an average crest propagation speed $\overline{C_{\text {crest }}}$ of $0.8 \mathrm{~m} \mathrm{~s}^{-1}$, found considering all wind/wave combinations is applied as a characteristic wave propagation speed in the PIV field-of-view. For all cases considered, the average crest propagation speed found deviated less than $10 \%$ from this value.

Figure 10 presents the velocity field of four individual waves, considering a range of wind speeds and incoming wave steepnesses. Where the shear layer separates over the leeward side of the crest (Fig. 10 a, b, d), there is a region of low velocity air, which is sheltered from the wind by the wave crest. The velocity vectors where the horizontal velocity is lower than the characteristic crest propagation speed $\overline{C_{\text {crest }}}$ are coloured in white. This area is considered as 'sheltered' and denoted $A_{\text {sheltered }}$. While the wave propagation speed is not a constant for the waves considered, applying a single threshold to define $A_{\text {sheltered }}$ provides consistency in the reported values, and it is clear that, e.g., an increase in the sheltered area is associated with a reduction in the airflow speed, not with an increase of the crest propagation speed used to evaluate the area. It can be seen that the area bounded by the separated shear layer and the interface, and the 'sheltered area' are closely related, although some discrepancies are seen, and the area bounded by the maximum vorticity line (not shown explicitly) is seen to be somewhat larger than the area where $u<\overline{C_{\text {crest }}}$. Determination of the separating streamline as discussed by Reul et al. (2008) was not always easy, especially for the highest wind case where the shear layer was seen to break up into a highly chaotic pattern some distance downstream of the crest. For this reason, and as they are seen to be well correlated, we focus on the development of $A_{\text {sheltered }}$. It is noted that when the high-intensity shear layer remained attached to the wave profile (such as Fig. 10c, no, or only a few, velocity vectors with $u<\overline{C_{\text {crest }}}$ were observed. The sheltered area is clearly related to the instantaneous crest height $\eta_{c}$, hence we use $A^{*}=A_{\text {sheltered }} / \eta_{c}^{2}$ as a normalized measure of the sheltering.

It should be noted that non-zero values of $A^{*}$ are not directly related to airflow separation. Owing to the no-slip condition at the surface, there will (considering a non-breaking wave) always be a region near the surface where the air velocity is lower than the propagation speed of the wave. It is also well known that the action of Reynolds stresses on the leeward side of the wave cause a thickening of the boundary layer and reduction of the wind velocity in this region. This effect is known as 'non-separated sheltering' (Belcher and Hunt 1998; Belcher 1999).

For each of the 13 experimental cases considered, 15 individual waves were analyzed as they entered the PIV field-of-view. A manual detection of the interface (evaluating also the instantaneous $\varepsilon_{\text {crest }}$ ) and PIV evaluation of the velocity field was performed, and $A^{*}$ was found for each wave. Based on visual characteristics, each wave was categorized as either 'breaking' or 'non-breaking', indicating whether the waves were assessed to be in a state of active breaking at the time of the PIV acquisition. Also the image prior to/after the image 


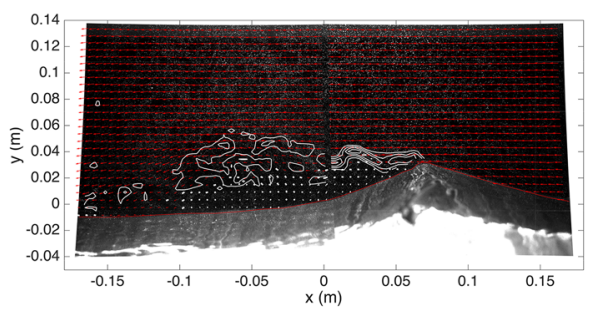

(a)

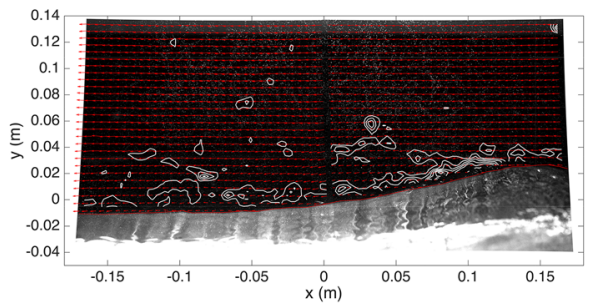

(c)

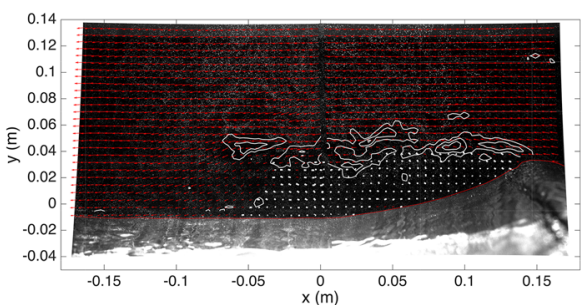

(b)

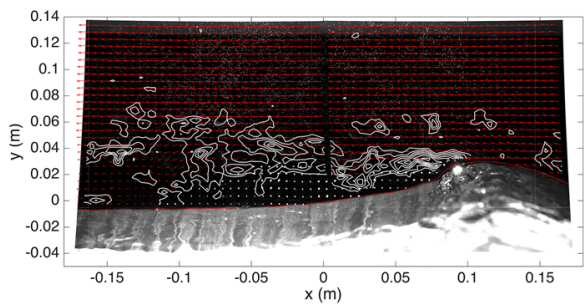

(d)

Fig. 10 Instantaneous velocity profiles overlaid raw PIV images. Red vectors: Horizontal velocity $>\overline{C_{\text {crest }}}$. White vectors: Horizontal velocity $<\overline{C_{\text {crest }}}$. Showing $1 / 2$ vectors. White contours: Contours of positive vorticity, separated by $100 \mathrm{~s}^{-1}$. a Wind case 1 , wave case 4 . b wind case 1 , wave case 5 . c wind case 5 , wave case 2 . $\mathbf{d}$ wind case 5 , wave case 2

used in the analysis was evaluated to categorize the breaking state. For the majority of the waves considered, it is assessed that the waves could be categorized as either breaking/nonbreaking with fair certainty, as a rough/spilling interface on the leeward side of the crest was clearly identified for the breaking waves. However, some waves were difficult to assess, and categorized as 'unknown state'. It should be noted that the waves assessed as 'nonbreaking' at the time of the PIV acquisition may have been close to the point of breaking, and transitioned to active breaking soon after the PIV acquisition.

In Figs. 11 and 12, scatter plots of $A^{*}$ vs. the crest front-face steepness $\varepsilon_{\text {crest }}$ or the Reynolds number based on the crest height $\left(R e_{\eta_{c}}=U_{\max } \eta_{c} / v_{\text {air }}\right.$, where $U_{\max }$ is the peak mean air speed, recorded without the influence of paddle-generated waves) are presented. The scatter points are coloured according to the evaluated breaking state. Red icons are assessed to be in a state of 'active breaking', green icons are 'non-breaking', and blue icons are 'unknown'.

Figure 11 presents $A^{*}$ vs. $\varepsilon_{\text {crest }}$ for each of the three wind speeds employed separately, while a collection of all experimental cases is presented in Fig. 12b. Although there is significant scatter in the experimental data, a correlation between the normalized sheltered area and the crest front-face steepness is observed, similar to the results by Reul et al. (2008) and Vollestad et al. (2019). While it is observed that the waves undergoing breaking are on average steeper than the observed non-breaking waves, it cannot be concluded that non-breaking or breaking waves at similar steepness induce a larger sheltered area based on these results.

Figure 12a presents $A^{*}$ vs. the wave Reynolds number. As $R e_{\eta_{c}}$ is increased above approximately $1 \times 10^{4}$, the peak values of $A^{*}$ observed are seen to stagnate. Reul et al. (2008) found that while the length of the separated region increased with $R e_{\eta_{c}}$ (considering $R e_{\eta_{c}}$ up to $3 \times 10^{4}$ ), the height of the separated shear layer was reduced as $R e_{\eta_{c}}$ was increased above approximately $2 \times 10^{4}$. While we consider slightly lower wave Reynolds numbers, a simi- 


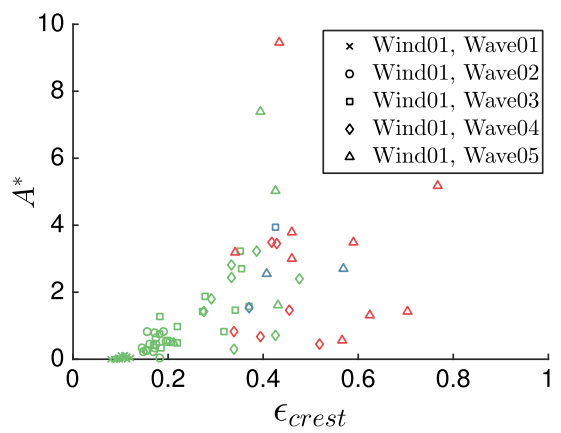

(a)

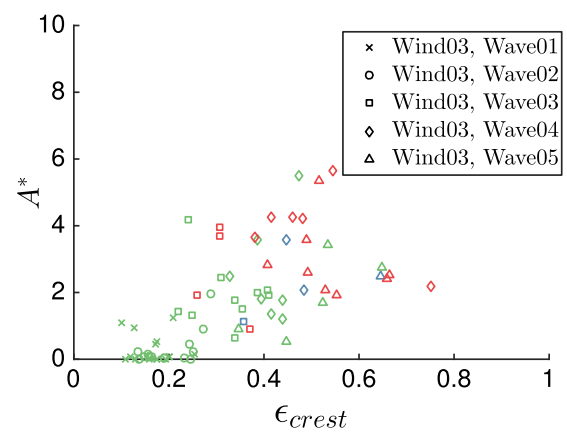

(b)

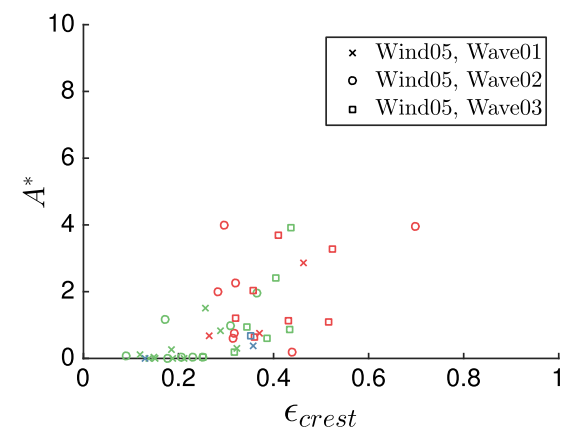

(c)

Fig. 11 Normalized sheltering for the wind cases considered. Green symbols: non-breaking wave. Red symbols: breaking waves. Blue symbols: wave not classified as either breaking or non-breaking. a wind case 1 . b wind case 3 . $\mathbf{c}$ wind case 5

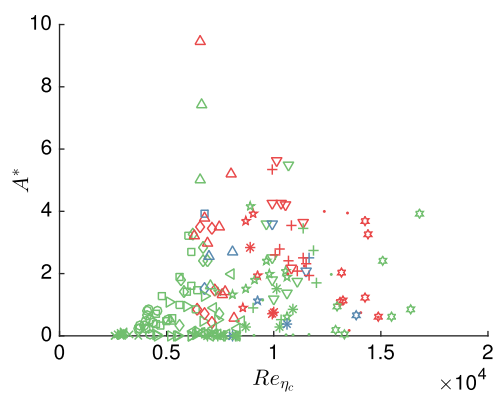

(a)

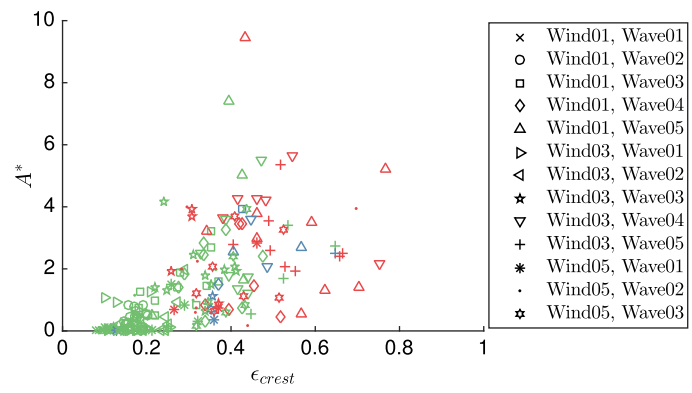

(b)

Fig. 12 Normalized sheltering vs $\operatorname{Re}_{\eta_{c}}$ (a) and $\varepsilon_{\text {crest }}$ (b) for all experimental cases. Green symbols: nonbreaking waves. Red symbols: breaking waves. Blue symbol: wave not classified as either breaking or nonbreaking

lar effect (reduction of the height of the separated shear layer with increasing wind speed) was inferred qualitatively from the experimental results, comparing the same incoming wave amplitude with increasing wind speeds. A larger range of $R e_{\eta_{c}}$ would be required to see if the trend holds, but the results can be seen in connection with the observations by Reul et al. (2008), that at sufficiently high wind speeds the height of the separated region is restricted. 


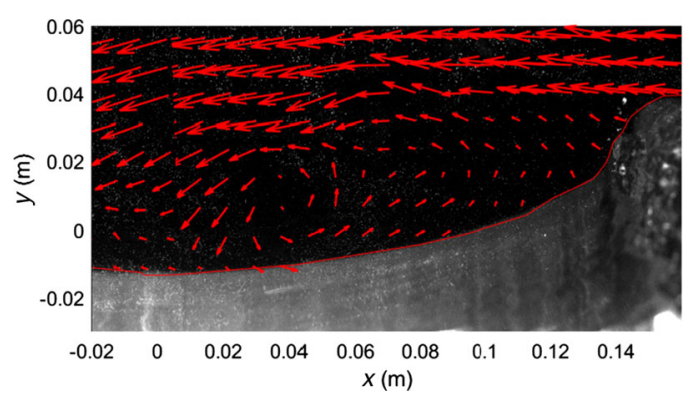

(a)

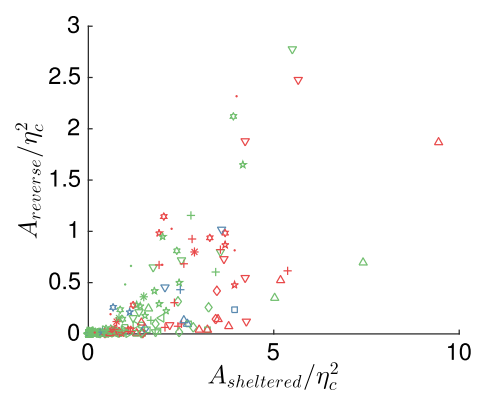

(b)

Fig. 13 a instantaneous vector field showing $1 / 3$ vectors. b scatter plot of $A_{\text {sheltered }} / \eta_{c}^{2}$ vs. $A_{\text {reverse }} / \eta_{c}^{2}$. The legends presented in Fig. 12 apply to the dots in plot b

The detachment of the shear layer and a significant sheltered region was typically accompanied by a distinct reverse flow region (in a fixed frame of reference) close to the interface. An example of such a reverse flow is presented in Fig. 13a. The extent and position of this region varied considerably between the instantaneous velocity fields. The area of reverse flow, $A_{\text {reverse }}$, was evaluated and normalized by the crest height $\eta_{c}$. Figure $13 \mathrm{~b}$ presents scatter plots of the normalized reverse flow area vs. the normalized sheltered area. Considerable scatter is observed in the data, and while the area of reverse flow is typically seen to represent $\approx 20 \%$ of the sheltered area, cases where the fraction is over $50 \%$ are also observed. There are also cases where the airflow is significantly sheltered, with very little or no signs of reverse flow. This could be related to the distinction between the 'non-separated' sheltering mechanism of Belcher (1999) and sheltering due to intermittent airflow separation. We note that similar to the results for $A^{*}$, there is little correlation between the state of breaking and the area of reverse flow observed.

To further analyze the flow in the sheltered region, the horizontal velocity (for all wind/wave cases) is recorded and organized according to the instantaneous $\varepsilon_{\text {crest }}$ of the relevant wave. Horizontal velocities of individual vectors $(u)$ within the sheltered region, and $\varepsilon_{\text {crest }}$ are organized within bins of $0.2 \mathrm{~m} \mathrm{~s}^{-1}$ and 0.1 respectively, and the probability of occurrence for a given horizontal velocity, given a wave within a specific $\varepsilon_{\text {crest }}$-interval $\left(P\left(u \mid \varepsilon_{\text {crest }}\right)\right)$ is evaluated for breaking and non-breaking waves individually; $\varepsilon_{\text {crest }}$-intervals with less than 1000 velocity vectors are omitted from the analysis. Results are presented in Fig. 14 as a three-dimensional scatter/surface plot (a) and as a two-dimensional scatter plot (b). Note that the wind and waves are directed in the negative $x$-direction, hence $u>0$ indicates reverse flow.

Results presented in Fig. 14 illustrate that in the region where a sufficient number of velocity vectors associated with both breaking and non-breaking waves are observed $(0.3<$ $\left.\varepsilon_{\text {crest }}<0.6\right)$, there is a significant overlap in the horizontal velocity probability functions (best observed in Fig. 14 a). This indicates that considering waves at a given crest frontface steepness, active wave breaking does not significantly modify the airflow structure in the sheltered region. It can also be noted that while the extent of the sheltered region was generally found to increase with increasing $\varepsilon_{\text {crest }}$ (ref. Figs. 11 and 12), the probability of reverse flow within these regions is seen to be smallest at the two largest $\varepsilon_{\text {crest }}$-intervals. A possible explanation for this observation is that the spilling motion of these steep, actively breaking waves act to reduce the reversed flow region, as water droplets are 'jetted' from the crest, creating a localized high-pressure zone on the leeward side of the crest. High-frequency, 


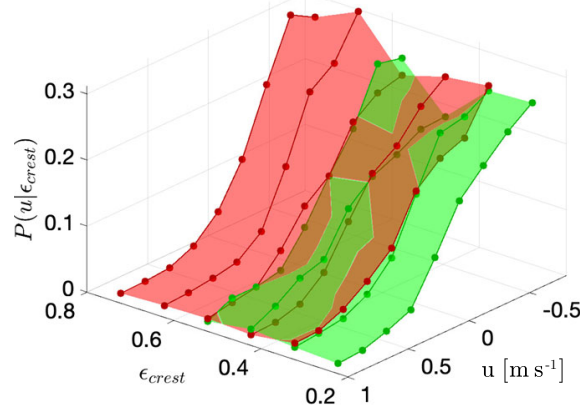

(a)

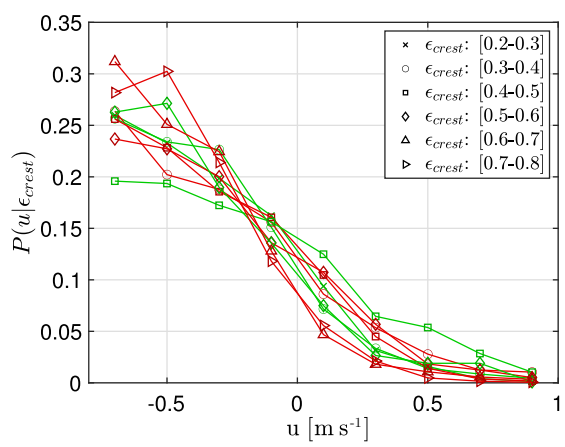

(b)

Fig. $14 P\left(u \mid \varepsilon_{\text {crest }}\right)$ (scatter points provide value) for non-breaking (green) and breaking (red) waves. Lines between each scatter point for a given $\varepsilon_{\text {crest }}$-interval (a and $\left.\mathbf{b}\right)$, and surface connecting the points (a) illustrate trends in the dataset

time-resolved PIV measurements of waves transitioning to this highly chaotic breaking state could shed some light onto these results.

\section{Discussion and Concluding Remarks}

The development of airflow above steep and breaking waves represents a significant challenge in our understanding of air-sea interactions, and the coupling between wave breaking and airflow separation is still an unresolved problem. In the present work, the waves are breaking due to a reduced water level at the step. The transition from deep water to intermediate depth is well known to induce wave breaking, and the dynamics of this breaking process will be somewhat different to wave breaking in the open ocean. Hence, also the impact of the breaking process on the overlying airflow can be expected to deviate somewhat from that observed over breaking waves in the open ocean. It is still assessed that the results presented can shed some light on the role of active wave breaking in modifying the airflow structure, and indirectly the form drag, above small-scale breaking waves, which are frequent in the ocean.

The results presented in Sect. 4.2.2 (while insufficient for a full statistical analysis), indicate that the wave geometric properties, and in particular the instantaneous crest front-face steepness, to a significant degree governs the area of the wave which is sheltered from the wind, while the state of breaking is not seen to significantly alter the airflow (considering waves at similar $\varepsilon_{\text {crest }}$ ). The extent of the sheltered area (through non-separated or separated sheltering) is assessed to be important, as it is related to the presence/severity of airflow separation, and the enhanced form drag observed over steep and breaking waves. Further analysis should focus on measuring the simultaneous pressure and velocity field over individual wave profiles, to see if this presumed link is directly applicable.

While the present work categorizes the waves as either 'breaking' or 'non-breaking', the time-development of the breaking process is not considered in the statistical analysis presented. It is considered likely that some of the scatter obtained in the present results could be reduced, and that the distinction from non-breaking waves would be more evident if only waves at the same state of the breaking process were considered. 
Acknowledgements The authors wish to acknowledge the strategic research initiative EarthFlows at the Faculty of Mathematics and Natural Sciences at the University of Oslo. Laboratory Head Engineer O. Gundersen is gratefully acknowledged for the technical assistance he provided during the experimental work.

Funding Open access funding provided by University of Oslo (incl Oslo University Hospital).

Open Access This article is licensed under a Creative Commons Attribution 4.0 International License, which permits use, sharing, adaptation, distribution and reproduction in any medium or format, as long as you give appropriate credit to the original author(s) and the source, provide a link to the Creative Commons licence, and indicate if changes were made. The images or other third party material in this article are included in the article's Creative Commons licence, unless indicated otherwise in a credit line to the material. If material is not included in the article's Creative Commons licence and your intended use is not permitted by statutory regulation or exceeds the permitted use, you will need to obtain permission directly from the copyright holder. To view a copy of this licence, visit http://creativecommons.org/licenses/by/4.0/.

\section{References}

Babanin AV, Banner ML, Young IR, Donelan MA (2007) Wave-follower field measurements of the wind-input spectral function. part iii: parameterization of the wind-input enhancement due to wave breaking. J Phys Oceanogr 37(11):2764-2775

Banner ML (1990) The influence of wave breaking on the surface pressure distribution in wind-wave interactions. J Fluid Mech 211:463-495

Banner ML, Melville WK (1976) On the separation of air flow over water waves. J Fluid Mech 77(04):825-842

Banner ML, Phillips OM (1974) On the incipient breaking of small scale waves. J Fluid Mech 65(4):647-656

Belcher SE (1999) Wave growth by non-separated sheltering. Eur J Mech B Fluids 18(3):447-462

Belcher SE, Hunt JCR (1998) Turbulent flow over hills and waves. Ann Rev Fluid Mech 30(1):507-538

Blenkinsopp CE, Chaplin JR (2008) The effect of relative crest submergence on wave breaking over submerged slopes. Coast Eng 55(12):967-974

Buckley MP, Veron F (2016) Structure of the airflow above surface waves. J Phys Oceanogr 46(5):1377-1397

Buckley MP, Veron F (2019) The turbulent airflow over wind generated surface waves. Eur J Mech B Fluids 73:132-143

Donelan MA, Babanin AV, Young IR, Banner ML (2006) Wave-follower field measurements of the wind-input spectral function. part ii: parameterization of the wind input. J Phys Oceanogr 36(8):1672-1689

Galvin JCJ (1968) Breaker type classification on three laboratory beaches. J Geophys Res 73(12):3651-3659

Gent PR, Taylor PA (1977) A note on ‘separation' over short wind waves. Boundary-Layer Meteorol 11(1):6587

Grue J, Clamond D, Huseby M, Jensen A (2003) Kinematics of extreme waves in deep water. Appl Ocean Res 25(6):355-366

Hinze JO (1967) Secondary currents in wall turbulence. Phys Fluids 10(9):S122-S125

Hinze JO (1973) Experimental investigation on secondary currents in the turbulent flow through a straight conduit. Appl Sci Res 28(1):453-465

Husain NT, Hara T, Buckley MP, Yousefi K, Veron F, Sullivan PP (2019) Boundary layer turbulence over surface waves in a strongly forced condition: LES and observation. J Phys Oceanogr 49(8):1997-2015

Johnson HK (2006) Wave modelling in the vicinity of submerged breakwaters. Coast Eng 53(1):39-48

Kawai S (1981) Visualization of airflow separation over wind-wave crests under moderate wind. BoundaryLayer Meteorol 21(1):93-104

Kihara N, Hanazaki H, Mizuya T, Ueda H (2007) Relationship between airflow at the critical height and momentum transfer to the traveling waves. Phys Fluids 19(1):015102

Kudryavtsev VN, Makin VK (2001) The impact of air-flow separation on the drag of the sea surface. BoundaryLayer Meteorol 98(1):155-171

Miron P, Vétel J (2015) Towards the detection of moving separation in unsteady flows. J Fluid Mech 779:819_ 841

Prandtl L (1927) Turbulent flow. In: International congress for applied mechanics, Zurich 1926 (also as N.A.C.A. Tech Memo 435)

Reul N, Branger H, Giovanangeli JP (2008) Air flow structure over short-gravity breaking water waves. Boundary-Layer Meteorol 126(3):477-505

Simpson RL (1989) Turbulent boundary-layer separation. Ann Rev Fluid Mech 21(1):205-232 
Smith ER, Kraus NC (1990) Laboratory study on macro-features of wave breaking over bars and artificial reefs. Coastal Engineering Research Center Vicksburg Ms. Technical report

Smith ER, Kraus NC (1991) Laboratory study of wave-breaking over bars and artificial reefs. J Waterway Port Coast Ocean Eng 117(4):307-325

Speziale CG (1982) On turbulent secondary flows in pipes of noncircular cross-section. Int J Eng Sci 20(7):863872

Sullivan PP, McWilliams JC (2010) Dynamics of winds and currents coupled to surface waves. Ann Rev Fluid Mech 42

Sullivan PP, Banner ML, Morison RP, Peirson WL (2018) Turbulent flow over steep steady and unsteady waves under strong wind forcing. J Phys Oceanogr 48(1):3-27

Tian Z, Perlin M, Choi W (2010) Observation of the occurrence of air flow separation over water waves. In: ASME 2010 29th international conference on ocean, offshore and arctic engineering. American Society of Mechanical Engineers, pp 333-341

Vollestad P, Ayati AA, Jensen A (2019) Experimental investigation of intermittent airflow separation and microscale wave breaking in wavy two-phase pipe flow. J Fluid Mech 878:796-819

Weissman MA (1986) Observations and measurements of air flow over water waves. In: Wave dynamics and radio probing of the ocean surface. Springer, pp 335-352

Yang Z, Deng BQ, Shen L (2018) Direct numerical simulation of wind turbulence over breaking waves. J Fluid Mech 850:120-155

Yao Y, Huang Z, Monismith SG, Lo EYM (2013) Characteristics of monochromatic waves breaking over fringing reefs. J Coast Res 29(1):94-104

Young IR (1999) Wind generated ocean waves. Elsevier

Publisher's Note Springer Nature remains neutral with regard to jurisdictional claims in published maps and institutional affiliations. 